\title{
A efetividade do treinamento auditivo formal em idosos usuários de próteses auditivas no período de aclimatização
}

\author{
Formal auditory training efficiency in elderly during the \\ acclimatization period
}

\author{
Elisiane de Crestani Miranda ${ }^{1}$, Adriana Neves de Andrade ${ }^{2}$, Daniela Gil ${ }^{3}$, Maria Cecília Martinelli Iório ${ }^{4}$
}

\begin{abstract}
RESUMO
Objetivo: Verificar a efetividade de um programa de treinamento auditivo formal em idosos usuários de próteses auditivas intraaurais no período de aclimatização. Métodos: A amostra foi composta por 18 idosos (idade média: 71, 38 anos), de ambos os sexos, adaptados há uma semana com próteses auditivas intra-aurais binaurais. Os participantes foram randomizados em dois grupos: Grupo Experimental (submetidos ao treinamento auditivo) e Grupo Controle (não submetidos ao treinamento auditivo). O Grupo Experimental participou de sete sessões de treinamento auditivo em cabina acústica, uma sessão por semana, com duração de 50 minutos cada. Os procedimentos de avaliação incluíram testes de reconhecimento de fala e questionário de auto-avaliação do handicap auditivo. Estes foram aplicados em duas oportunidades, antes ( $1^{\mathrm{a}}$ avaliação) e depois ( $2^{\mathrm{a}}$ avaliação) do treinamento auditivo no Grupo Experimental e na avaliação inicial e final do estudo no Grupo Controle. Resultados: No Grupo Experimental, o Índice de Reconhecimento de Fala e Fala com Ruído Branco foram significantemente melhores após o treinamento auditivo (2a avaliação). Já o estudo das relações sinal/ruído no teste de reconhecimento de sentenças no ruído revelou uma tendência (p-valor próximo a 0,05) de melhora na avaliação pós-treinamento. Observou-se nos idosos do Grupo Experimental que os resultados obtidos na $2^{\mathrm{a}}$ avaliação não foram significantemente melhores aos obtidos no Grupo Controle em todos os testes. Conclusão: Pode-se concluir que um programa de reabilitação aural, incluindo treinamento auditivo formal beneficia os idosos no período de adaptação das próteses auditivas, bem como modifica o comportamento auditivo destes indivíduos.
\end{abstract}

Descritores: Perda auditiva/reabilitação; Implantes cocleares; Auxiliares de audição; Adaptação fisiológica/fisiologia; Idoso

\section{INTRODUÇÃO}

A audição é fundamental na interação entre seres humanos. O sistema auditivo em envelhecimento geralmente leva a uma perda na sensibilidade do limiar e uma redução na habilidade de compreender a fala em níveis confortáveis ${ }^{(1-2)}$.

Pesquisa de mestrado financiada pela Fundação de Amparo a Pesquisa do Estado de São Paulo - FAPESP, realizada na Universidade Federal de São Paulo - UNIFESP - São Paulo (SP), Brasil. Trabalho recebeu Prêmio de Menção Honrosa no XXII Encontro Internacional de Audiologia - EIA, Natal (RN), 2007.

(1) Mestre, Fonoaudióloga do Núcleo Integrado de Atendimento, Pesquisa e Ensino em Audição da Universidade Federal de São Paulo - UNIFESP - São Paulo (SP), Brasil.

(2) Pós-graduanda em Distúrbios da Comunicação Humana pela Universidade Federal de São Paulo - UNIFESP - São Paulo (SP), Brasil.

(3) Doutora, Professor Adjunto do Curso de Fonoaudiologia da Universidade Federal de São Paulo - UNIFESP - São Paulo (SP), Brasil.

(4) Livre-Docente, Professor Adjunto do Curso de Fonoaudiologia da Universidade Federal de São Paulo - UNIFESP - São Paulo (SP), Brasil.

Endereço para correspondência: Elisiane Crestani de Miranda. R. Borges Lagoa, 512/92B, Vila Clementino, São Paulo - SP, CEP 04038-000. E-mail: lisi_miranda@hotmail.com

Recebido em: 20/7/2007; Aceito em: 30/11/2007
A deficiência auditiva na população idosa é uma das três condições crônicas mais prevalentes, ficando atrás somente da artrite e da hipertensão.

Uma forma de minimizar os efeitos negativos da deficiência auditiva em idosos é a utilização dos recursos tecnológicos disponíveis, ou seja, dos aparelhos de amplificação sonora individual (AASI). Entretanto, diversos fatores podem determinar a não-utilização desses instrumentos, como: a falta de orientação ao usuário; falta de aconselhamento; e a criação de expectativas inadequadas, relacionadas aos benefícios; além de possíveis limitações dos próprios instrumentos ${ }^{(3)}$.

$\mathrm{O}$ termo aclimatização refere-se à melhora no reconhecimento de fala ao longo do tempo, em decorrência da introdução da amplificação, podendo variar de seis a 12 semanas de uso da amplificação e pode representar uma melhora entre zero e $10 \%$ no reconhecimento de fala ${ }^{(4-5)}$.

No entanto, não há um consenso sobre a existência da aclimatização, ou seja, se adultos usuários de próteses auditivas aprendem espontaneamente a utilizar as novas informações acústicas. Algumas pesquisas demonstraram a existência do efeito de aclimatização ${ }^{(4,6-8)}$. Já outros estudos mostraram que tais efeitos são mínimos ou inexistentes ${ }^{(9-12)}$. 
Embora a audibilidade de um sinal de fala possa ser restaurada imediatamente, após a adaptação de próteses auditivas, o usuário necessita de um tempo para aprender a interpretar as informações acústicas introduzidas recentemente. Treinar o indivíduo, que está recebendo o sinal de fala amplificado, pode ser necessário para otimizar os benefícios das próteses auditivas.

O treinamento pode ser capaz de acelerar o processo de aclimatização e de aprimorar as habilidades de reconhecimento de fala. A combinação do treinamento com a amplificação pode produzir um pequeno, porém significante, benefício em tempo menor do que 12 a 18 semanas $^{(13)}$.

Recomenda-se utilizar algum tipo de treinamento auditivo, formal ou informal, após a adaptação das próteses auditivas, abordando o novo sinal amplificado e especialmente o novo padrão de processamento. A autora afirma que a percepção de sons de fala pode ser modificada diretamente pelo treinamento auditivo ${ }^{(14)}$.

A realização deste estudo partiu do questionamento se idosos novos usuários de próteses auditivas poderiam se beneficiar do Treinamento Auditivo Formal (TAF). Apesar de saber-se que, nas primeiras semanas de uso das próteses auditivas, os pacientes estão em período de adaptação/ajustamento às mesmas, estudou-se um grupo de idosos com uma semana de uso de amplificação, a fim de verificar se o TAF otimizaria os resultados da adaptação e/ou aclimatização.

O objetivo deste estudo foi verificar a efetividade de um programa de treinamento auditivo formal em idosos, no período de aclimatização.

\section{MÉTODOS}

Este estudo foi realizado no Núcleo Integrado de Assistência, Ensino e Pesquisa em Audição (NIAPEA) da Universidade Federal de São Paulo (UNIFESP), após aprovação da Comissão de Ética em Pesquisa da UNIFESP, com o número CEP 1801/06.

Foram considerados critérios de inclusão os seguintes aspectos: perda auditiva neurossensorial simétrica de grau de grau leve a moderadamente severo bilateral ${ }^{(15)}$, de configuração plana ou levemente descendente nas freqüências altas, índice de reconhecimento de fala igual ou superior à $72 \%$ bilateralmente, indicação de próteses auditivas intraaurais em adaptação binaural e idade acima de 60 anos.
Para seleção da amostra foi analisada a lista de pacientes que aguardavam a concessão de próteses auditivas no Núcleo Integrado de Assistência, Ensino e Pesquisa em Audição - NIAPEA. Foram encontrados 44 idosos e a partir da análise dos prontuários foram excluídos 22 indivíduos, dos quais 12 pacientes com reconhecimento de fala inferior a $72 \%$, dois com comprometimento cognitivo e oito residiam fora do estado de São Paulo. Dessa forma, no momento da concessão das próteses auditivas, os 22 indivíduos selecionados nesta primeira análise foram convidados a participar da pesquisa. No entanto, dois indivíduos não se dispuseram a participar e outros dois abandonaram o estudo. Assim sendo, a amostra final contou com a participação de 18 indivíduos no período de aclimatização, sendo 11 do sexo feminino e sete do sexo masculino, com faixa etária variando entre 61 e 83 anos (média: 71,38 anos).

O próprio indivíduo realizou um sorteio o qual iria incluí-lo no Grupo Experimental (submetidos ao treinamento auditivo) ou no Grupo Controle (não submetidos ao treinamento auditivo).

Nos Quadros 1 e 2 são caracterizados os integrantes do Grupo Experimental e Grupo Controle, respectivamente.

Os participantes do estudo foram submetidos à: Audiometria Tonal com próteses auditivas com fones auriculares, pesquisa da relação sinal/ruído (relação S/R) obtida no teste de Reconhecimento de Sentenças no Ruído (LRSR), pesquisa do Índice de Reconhecimento de Fala não Sensibilizado (IPRF com gravação), o teste de Fala com Ruído Branco (FRB) e questionário de auto-avaliação do handicap auditivo (Hearing Handicap Inventory for the Elderly HHIE). O tempo de duração da avaliação foi cerca de 45 minutos. Todos os procedimentos de avaliação foram realizados com os idosos fazendo uso de suas próteses auditivas intra-aurais.

Os testes de avaliação foram aplicados em duas oportunidades, antes ( $1^{\mathrm{a}}$ avaliação) e depois ( $2^{\mathrm{a}}$ avaliação) do treinamento auditivo no Grupo Experimental e na avaliação inicial e final do estudo (oito semanas) no Grupo Controle, obedecendo ao mesmo intervalo de tempo entre as avaliações, as quais foram realizadas mediante convocação. As reavaliações foram realizadas por uma examinadora diferente da que aplicou a avaliação inicial, o que caracteriza um estudo do tipo duplo-cego, pois a mesma não tinha conhecimento se o indivíduo havia ou não recebido treinamento auditivo.

Quadro 1. Idade, sexo, nível de escolaridade, preferência manual, grau de perda auditiva dos idosos do grupo experimental (GE)

\begin{tabular}{|llllll|}
\hline Indivíduos & Idade & Sexo & Escolaridade & Preferência Manual & Grau da PANS \\
\hline 1 & 66 & $\mathrm{~F}$ & $2^{\circ}$ grau completo & Direita & Moderada \\
2 & 79 & $\mathrm{~F}$ & $1^{\circ}$ grau completo & Direita & Moderada \\
3 & 73 & $\mathrm{~F}$ & $1^{\circ}$ grau completo & Direita & Moderada \\
4 & 62 & $\mathrm{M}$ & $2^{\circ}$ grau completo & Direita & Moderada \\
5 & 61 & $\mathrm{M}$ & $1^{\circ}$ grau incompleto & Moderada \\
6 & 62 & $\mathrm{~F}$ & $1^{\circ}$ grau incompleto & Direita & Moderada \\
7 & 79 & $\mathrm{~F}$ & $3^{\circ}$ grau incompleto & Direita & Leve a Moderada \\
8 & 64 & $\mathrm{~F}$ & $1^{\circ}$ grau incompleto & Direita & Moderada \\
\hline
\end{tabular}

Legenda: F - Feminino; M - Masculino; PANS - Perda Auditiva Neurossensorial 
Quadro 2. Idade, sexo, nível de escolaridade, preferência manual, grau de perda auditiva dos idosos do grupo controle (GC)

\begin{tabular}{|c|c|c|c|c|c|}
\hline Indivíduos & Idade & Sexo & Escolaridade & Preferência Manual & Grau da PANS \\
\hline 1 & 72 & $\mathrm{M}$ & $1^{\circ}$ grau incompleto & Direita & Leve a Moderada \\
\hline 2 & 76 & $\mathrm{~F}$ & $1^{\circ}$ grau completo & Direita & Leve \\
\hline 3 & 73 & $\mathrm{M}$ & $1^{\circ}$ grau incompleto & Direita & Leve a Moderada \\
\hline 4 & 76 & $\mathrm{~F}$ & $1^{\circ}$ grau incompleto & Direita & Moderada \\
\hline 5 & 70 & $\mathrm{M}$ & $1^{\circ}$ grau incompleto & Direita & Moderada \\
\hline 6 & 78 & $\mathrm{~F}$ & $1^{\circ}$ grau completo & Direita & Leve a Moderada \\
\hline 7 & 64 & $\mathrm{M}$ & $3^{\circ}$ grau incompleto & Direita & Moderada \\
\hline 8 & 68 & $\mathrm{~F}$ & $1^{\circ}$ grau incompleto & Direita & Leve a Moderada \\
\hline 9 & 79 & $\mathrm{M}$ & $1^{\circ}$ grau completo & Direita & Leve a Moderada \\
\hline 10 & 83 & $M$ & $1^{\circ}$ grau incompleto & Direita & Leve a Moderada \\
\hline
\end{tabular}

Legenda: F - Feminino; M - Masculino; PANS - Perda Auditiva Neurossensorial

O Treinamento Auditivo Formal (TAF) proposto neste estudo foi organizado em sete sessões, realizadas uma vez por semana, com duração de 50 minutos cada ${ }^{(16-17)}$. O material empregado para realização do treinamento auditivo foram os CDs integrantes do livro: Processamento Auditivo Central: Manual de Avaliaçãoo ${ }^{(18)}$.

As orelhas direita e esquerda foram treinadas separadamente, por meio de fones auriculares. Foi estabelecida uma intensidade fixa para a apresentação do estímulo (verbal ou não - verbal) e variou-se a intensidade do ruído competitivo. Da primeira à sexta sessão de treinamento, foram apresentadas tarefas de escuta monótica na presença de ruído competitivo, variando a relação sinal ruído de positiva (mais favorável) para negativa (menos favorável). A última sessão de treinamento envolveu tarefa de integração binaural.

O cronograma das sessões de treinamento auditivo bem como as atividades propostas em cada uma delas segue abaixo:

$1^{\text {a }}$ Sessão - Figura-fundo para sons verbais (frases) - Teste SSI - Orelha Direita

$2^{\text {a }}$ Sessão - Figura-fundo para sons verbais (frases) - Teste SSI - Orelha Esquerda

$3^{\text {a }}$ Sessão: Figura-fundo para sons verbais (dígitos) - Teste Dicótico de Dígitos - Orelha Direita

$4^{\text {a }}$ Sessão: Figura-fundo para sons verbais (dígitos) - Teste Dicótico de Dígitos - Orelha Esquerda

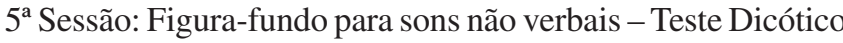
Não Verbal - Orelha Esquerda e Figura-fundo para sons verbais (dígitos) - Teste Dicótico de Dígitos - Orelha Direita.

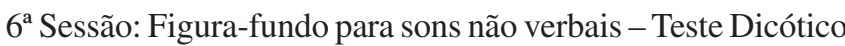
Não Verbal - Orelha Direita e Figura-fundo para sons verbais (dígitos) - Teste Dicótico de Dígitos - Orelha Esquerda.

$7^{\mathrm{a}}$ Sessão: Integração Binaural - para sons verbais (dígitos) -

Teste Dicótico de Digitos e para sons não verbais - Teste Dicótico Não - Verbal

Antes do início das sessões, os pacientes foram questionados sobre o número de horas diárias de utilização das próteses, e foram sempre estimulados a aumentar o tempo de uso. Além disso, foram reforçadas as orientações sobre a importância do uso das próteses auditivas também em ambientes de difícil escuta, pois muitos idosos referiam evitar estes locais ou retirar as próteses auditivas, por apresentarem insegurança e dificuldade na comunicação.

Ao término de cada sessão, foram expostos os objetivos das tarefas trabalhadas. Além disso, foram fornecidas orientações sobre estratégias de comunicação para facilitar a compreensão de fala, manter a qualidade do diálogo e desenvolver confiança ao interagir com outras pessoas. Os idosos foram estimulados a relatarem semanalmente as diferenças, dificuldades/facilidades percebidas durante o treinamento com o uso das próteses auditivas. Por outro lado, o profissional reforçava os pontos positivos do desempenho do paciente e desafiando-o a superar suas dificuldades na próxima sessão.

\section{RESULTADOS}

Inicialmente realizou-se o levantamento dos resultados dos testes IPRF, FRB, relação S/R-LRSR e o questionário HHIE entre as avaliações, nos Grupos: Experimental (com treinamento) e Controle (sem treinamento).

Para a comparação dos resultados entre as avaliações, foi utilizado o Teste T-Student Pareado.

Nas Tabelas 1 a 4 apresentamos os resultados dos testes IPRF, FRB, relações S/R - LRSR e HHIE dos Grupos Experimental e Controle para a $1^{\mathrm{a}}$ e a $2^{\mathrm{a}}$ avaliação.

Para verificar se o treinamento auditivo foi efetivo nos idosos no período de aclimatização, foram comparados os resultados obtidos nos indivíduos treinados (GE) e não treinados (GC) por meio o teste ANOVA.

Tabela 1. Medidas descritivas dos IPRF na $1^{\mathrm{a}}$ e $2^{\mathrm{a}}$ avaliação dos grupos $\mathrm{C}$ e $\mathrm{E}$, e resultados do teste T-Student Pareado.

\begin{tabular}{lcccc}
\hline IPFR & \multicolumn{2}{c}{ GC (sem treinamento) } & \multicolumn{2}{c}{ GE (com treinamento) } \\
& Aval 1 & Aval 2 & Aval 1 & Aval 2 \\
\hline Média & 78,60 & 75,30 & 75,25 & 78,50 \\
Mediana & 80 & 77 & 72 & 78 \\
Desvio Padrão & 5,70 & 7,66 & 4,67 & 4,59 \\
\hline p-valor & \multicolumn{2}{c}{$0,009^{*}$} & \multicolumn{2}{c}{$0,001^{*}$}
\end{tabular}

${ }^{*} p$-valor significante $<0,05(5 \%)$

Legenda: IPRF - Índice Perceptual de Reconhecimento de Fala; Aval $1-1^{\text {a }}$ avaliação; Aval 2 -2 ${ }^{\mathrm{a}}$ avaliação; GE - Grupo Experimental; GC - Grupo Controle 
Tabela 2. Medidas descritivas dos resultados do teste FRB na $1^{\mathrm{a}}$ e $2^{\mathrm{a}}$ avaliação dos grupos $\mathrm{C}$ e E, e resultados do teste T-Student Pareado.

\begin{tabular}{lcccc}
\hline FRB & \multicolumn{2}{c}{ GC (sem treinamento) } & \multicolumn{2}{c}{ GE (com treinamento) } \\
& Aval 1 & Aval 2 & Aval 1 & Aval 2 \\
\hline Média & 67,00 & 65,20 & 61,00 & 70,50 \\
Mediana & 70 & 70 & 62 & 70 \\
Desvio Padrão & 15,51 & 15,03 & 9,18 & 7,14 \\
\hline p-valor & \multicolumn{2}{c}{0,243} & \multicolumn{2}{c}{$<0,001^{*}$} \\
\hline
\end{tabular}

*p-valor significante $<0,05(5 \%)$

Legenda: FRB - Fala com Ruído Branco; Aval $1-1^{\mathrm{a}}$ avaliação; Aval $2-2^{\mathrm{a}}$ avaliação; GE - Grupo Experimental; GC - Grupo Controle

Tabela 3. Medidas descritivas das relações S/R do teste LRSR na $1^{a} \mathrm{e}$ $2^{\mathrm{a}}$ avaliação nos grupos $\mathrm{C}$ e E, e resultados do teste T-Student Pareado.

\begin{tabular}{lcccc}
\hline LRSR & \multicolumn{2}{c}{ GC (sem treinamento) } & \multicolumn{2}{c}{ GE (com treinamento) } \\
& Aval 1 & Aval 2 & Aval 1 & Aval 2 \\
\hline Média & 2,51 & 3,25 & 3,58 & 2,40 \\
Mediana & 2 & 3 & 3 & 3 \\
Desvio Padrão & 2,96 & 2,85 & 2,21 & 2,50 \\
\hline p-valor & \multicolumn{3}{c}{0,347} & \multicolumn{2}{c}{$0,075 \#$} \\
\hline
\end{tabular}

${ }^{*}$ - -valor significante $<0,05(5 \%)$

Legenda: LRSR - Limiar de Reconhecimento de Sentença no Ruído; Aval $1-1^{\text {a }}$ avaliação; Aval 2 - $2^{\mathrm{a}}$ avaliação; GE - Grupo Experimental; GC - Grupo Controle; \# próximos ao nível de significância mínima de 0,05 (5\%)

Tabela 4. Medidas descritivas do questionário HHIE na $1^{\mathrm{a}} \mathrm{e} 2^{\mathrm{a}}$ avaliação dos grupos $\mathrm{C}$ e $\mathrm{E}$, e resultados do teste T-Student Pareado.

\begin{tabular}{lcccc}
\hline HHIE & \multicolumn{2}{c}{ GC (sem treinamento) } & \multicolumn{2}{c}{ GE (com treinamento) } \\
& Aval 1 & Aval 2 & Aval 1 & Aval 2 \\
\hline Média & 21,11 & 17,33 & 23,75 & 8,75 \\
Mediana & 14 & 4 & 8 & 5 \\
Desvio Padrão & 19,52 & 21,35 & 24,36 & 11,21 \\
\hline p-valor & \multicolumn{3}{c}{0,131} & \multicolumn{2}{c}{$0,044^{*}$} \\
\hline
\end{tabular}

${ }^{*} \mathrm{p}$-valor significante $<0,05(5 \%)$

Legenda: HHIE - Hearing Handicap Inventory for the Elderly; Aval 1- $1^{\mathrm{a}}$ avaliação; Aval 2 - $2^{\mathrm{a}}$ avaliação; GE - Grupo Experimental; GC - Grupo Controle

A seguir, são apresentadas, nas Tabelas 5 a 8, o estudo comparativo entre os resultados dos testes IPRF, FRB e relação S/R - LRSR e HHIE obtidos nos Grupos Controle e Experimental.

Tabela 5. Medidas descritivas dos IPRF obtidas nos idosos dos grupos $\mathrm{C}$ e $\mathrm{E}$, e resultados do teste ANOVA ( $p$-valor).

\begin{tabular}{lcccc}
\hline Medidas & \multicolumn{2}{c}{ IPFR (1) } & \multicolumn{2}{c}{ IPFR (2) } \\
descritivas & GC & GE & GC & CE \\
\hline Média & 78,60 & 75,25 & 75,30 & 78,50 \\
Mediana & 80 & 72 & 77 & 78 \\
Desvio Padrão & 5,70 & 4,67 & 7,66 & 4,59 \\
\hline p-valor & \multicolumn{2}{c}{$0,067 \#$} & \multicolumn{2}{c}{0,150} \\
\hline
\end{tabular}

*p-valor significante $<0,05(5 \%)$

Legenda: IPRF - Índice Percentual de Reconhecimento de Fala; $1-1^{\mathrm{a}}$ avaliação; $2-2^{\mathrm{a}}$ avaliação; GE - Grupo Experimental; GC- Grupo Controle; \# próximos ao nível de significância mínima de 0,05 (5\%)
Tabela 6. Medidas descritivas do teste FRB obtidas nos idosos dos grupos $\mathrm{C}$ e E, e resultados do teste ANOVA ( $p$-valor).

\begin{tabular}{lcccc}
\hline Medidas & \multicolumn{2}{c}{ FRB (1) } & \multicolumn{2}{c}{ FRB (2) } \\
descritivas & GC & GE & GC & GE \\
\hline Média & 67,00 & 61,00 & 65,20 & 70,50 \\
Mediana & 70 & 62 & 70 & 70 \\
Desvio Padrão & 15,51 & 9,18 & 15,03 & 7,14 \\
\hline p-valor & \multicolumn{2}{c}{0,181} & \multicolumn{2}{c}{0,204} \\
\hline
\end{tabular}

${ }^{*} \mathrm{p}$-valor significante $<0,05(5 \%)$

Legenda: FRB - Fala com Ruído Branco; $1-1^{\mathrm{a}}$ avaliação; 2 - $2^{\mathrm{a}}$ avaliação; GE Grupo Experimental; GC - Grupo Controle

Tabela 7. Medidas descritivas das relações S/R do teste LRSR obtidas nos idosos dos grupos $\mathrm{C}$ e $\mathrm{E}$, e resultados do teste ANOVA (p-valor).

\begin{tabular}{lcccc}
\hline Medidas & \multicolumn{2}{c}{ LRSR (1) } & \multicolumn{2}{c}{ LRSR (2) } \\
descritivas & GC & GE & GC & GE \\
\hline Média & 2,51 & 3,58 & 3,25 & 2,40 \\
Mediana & 2 & 3 & 3 & 3 \\
Desvio Padrão & 2,96 & 2,21 & 2,85 & 2,50 \\
\hline p-valor & \multicolumn{3}{c}{0,241} & \multicolumn{3}{c}{0,352}
\end{tabular}

${ }^{*} \mathrm{p}$-valor significante $<0,05(5 \%)$

Legenda: LRSR - Limiar de Reconhecimento de Sentenças no Ruído; $1-1^{\text {a }}$ avaliação; 2 -2 ${ }^{\mathrm{a}}$ avaliação; GE - Grupo Experimental; GC - Grupo Controle

Tabela 8. Medidas descritivas dos resultados do HHIE obtidas nos idosos dos grupos $\mathrm{C}$ e E, e resultados do teste ANOVA (p-valor).

\begin{tabular}{lcccc}
\hline Medidas & \multicolumn{2}{c}{ HHIE (1) } & \multicolumn{2}{c}{ HHIE (2) } \\
descritivas & GC & GE & GC & CE \\
\hline Média & 22,00 & 23,75 & 19,00 & 8,75 \\
Mediana & 16 & 8 & 7 & 5 \\
Desvio Padrão & 20,67 & 24,36 & 22,19 & 11,21 \\
\hline p-valor & \multicolumn{3}{c}{0,878} & \multicolumn{3}{c}{0,247}
\end{tabular}

${ }^{*} \mathrm{p}$-valor significante $<0,05(5 \%)$

Legenda: HHIE - Hearing Handicap Inventory for the Elderly; GE - Grupo Experimental; GC - Grupo Controle; $1-1^{\mathrm{a}}$ avaliação; 2 - $2^{\mathrm{a}}$ avaliação; 3 - $3^{\mathrm{a}}$ avaliação

\section{DISCUSSÃO}

A análise dos resultados dos testes IPRF e FRB dos idosos do Grupo Experimental (GE) revelou um aumento significante na porcentagem de acertos após o treinamento auditivo ( $2^{\mathrm{a}}$ avaliação), sendo que estes valores aproximaram-se dos encontrados em idosos com audição dentro do padrão da normalidade (78,50\% no IPRF e 70,50\% no FRB). Já o estudo das relações $S / R$ no teste de reconhecimento de sentenças no ruído relevou uma tendência (p-valor próximo a 0,05 ) de melhora na avaliação pós-treinamento (Tabelas 1 , 2 e 3 ).

Estes dados demonstraram que a estimulação das habilidades auditivas por meio do TAF promoveu melhora no reconhecimento de fala.

Os idosos com perda auditiva são capazes de melhorar significativamente sua capacidade de reconhecimento de fala por meio do treinamento auditivo ${ }^{(19)}$. $\mathrm{O}$ treinamento pode ser 
capaz de acelerar o processo de aclimatização e de aprimorar as habilidades de reconhecimento de fala. A combinação do treinamento com a amplificação pode produzir um pequeno, porém significante, benefício em tempo menor do que 12 a 18 semanas, como o preconizado pelos autores que estudam aclimatização ${ }^{(13)}$.

A reabilitação auditiva não deve envolver somente a compensação da alteração periférica, mas também deve superar os efeitos centrais da privação sensorial auditiva ${ }^{(20)}$. Dessa forma, a reabilitação com a adaptação de próteses auditivas deve aumentar o número de pacientes satisfeitos com suas próteses auditivas. Neste cenário, um programa de TAF tem papel fundamental de maximizar os benefícios oferecidos pela amplificação ${ }^{(21)}$.

A avaliação subjetiva por meio da análise do questionário HHIE mostrou uma redução significante da percepção do handicap auditivo após o treinamento ( $2^{\mathrm{a}}$ avaliação). Estes valores foram de $23,75 \%$ na $1^{a}$ avaliação (pré-treinamento) para $8,75 \%$ na $2^{\mathrm{a}}$ (pós-treinamento) (Tabela 4).

$\mathrm{O}$ treinamento auditivo é um procedimento de reabilitação efetivo para idosos com perda auditiva, melhorando de forma significante tanto a discriminação de fala como o funcionamento psicossocial ${ }^{(22)}$.

Dessa forma, os dados apresentados sugerem que o programa de treinamento auditivo em cabina acústica foi efetivo em idosos que se encontram no processo de adaptação das próteses auditivas, ou seja, durante a aclimatização. Os resultados do Grupo Experimental mostraram melhora tanto no desempenho nos testes comportamentais quanto na avaliação subjetiva.

No Grupo Controle observou-se que não houve mudança no desempenho entre a $1^{\mathrm{a}}$ e $2^{\mathrm{a}}$ avaliação nos testes FRB, relação S/R - LRSR e HHIE durante o período estudado. No entanto, o mesmo não foi observado no teste IPRF que apresentou deterioração significante de desempenho (de 78,60\% para $75,30 \%$ acertos) após as oito semanas de uso das próteses auditivas.

Além da significante piora do IPRF médio, encontrada no período de estudo, foi observado que os demais testes realizados apresentaram uma discreta piora dos resultados na avaliação final ( $2^{\mathrm{a}}$ avaliação). Estes achados podem estar relacionados às dificuldades vivenciadas nas primeiras semanas de uso da amplificação, sendo que muitos pacientes do Grupo Controle relataram não fazer uso integral de suas próteses auditivas neste período. Diferentemente do Grupo Experimental, os idosos que não participaram do TAF (Grupo Controle) não receberam acompanhamento e aconselhamento semanal, o que pode ter minimizado as dificuldades encontradas nas primeiras semanas de uso da amplificação.

$\mathrm{O}$ efeito da aclimatização propriamente dito, não foi objetivo deste estudo; no entanto, os idosos do GC foram acompanhados desde o momento da adaptação das próteses auditivas até a oitava semana de uso. A partir dos resultados dos testes realizados, observou-se que não houve melhora no reconhecimento de fala nas primeiras semanas de uso da amplificação; pelo contrário houve uma pequena redução desta habilidade. Dessa forma, neste estudo não foi encontrada evidência da existência de aclimatização, como também observado em outros estudos ${ }^{(7,9-12)}$.

Conforme pesquisas realizadas investigando a plasticidade neural, a organização do Sistema Nervoso Auditivo Central (SNAC) pode mudar entre a instalação da perda auditiva e o momento quando são adaptadas as próteses auditivas. As próteses auditivas deveriam ser adaptadas antes que o sistema nervoso tivesse se reorganizado e a oportunidade para otimizar a protetização tenha ocorrido ${ }^{(23)}$. As próteses auditivas compensam parcialmente as dificuldades decorrentes da deficiência auditiva e devido a isso muitos pacientes rejeitam o uso das próteses auditivas e alguns usuários não estão completamente satisfeitos com as mesmas ${ }^{(20)}$.

No estudo comparativo entre Grupo Controle e Experimental verificou-se na $1^{\mathrm{a}}$ avaliação (inicial) que os idosos do Grupo Experimental apresentaram um pior desempenho (pvalor próximo a 0,05 ) do que os idosos do Grupo Controle somente no teste IPRF, respectivamente $75,25 \%$ e $78,60 \%$ de acertos.

$\mathrm{Na} 2^{\mathrm{a}}$ avaliação (com oito semanas com ou sem TAF) observou-se que os resultados obtidos no Grupo Experimental não foram significantemente melhores aos obtidos no Grupo Controle em todos os testes (IPRF, FRB, relação S/R - LRSR e HHIE) (Tabelas 5 a 8).

A partir desses dados, depreende-se que a diferença observada na $1^{\mathrm{a}}$ avaliação entre grupos no teste IPRF deixa de existir, demonstrando que os idosos do Grupo Experimental, após treinamento, apresentaram melhora no seu desempenho, o que já foi demonstrado no estudo entre as avaliações.

Além dos resultados apresentados anteriormente, vale ressaltar que, embora não significante, nos idosos do Grupo Controle, observou-se uma discreta piora nos resultados obtidos na $2^{\mathrm{a}}$ avaliação ( $8^{\mathrm{a}}$ semana de uso das próteses auditivas) em todos os testes. Isto sugere que apenas a audibilidade fornecida pelas próteses auditivas aos idosos do Grupo Controle não foi suficiente para promover a adequação da habilidade de reconhecimento de fala.

Para modificar o processamento de um sinal devemos utilizar amplificação e, posteriormente, refinarmos as habilidades de processamento da informação ${ }^{(24)}$.

No que se refere aos efeitos sociais e emocionais avaliados por meio do questionário HHIE, verificou-se que houve uma redução da percepção do handicap em ambos os grupos (Tabela 8). No entanto, os idosos do Grupo Controle permaneceram com o grau de percepção leve a moderado $(22 \%$ para 19\%) e os idosos o Grupo Experimental (com treinamento) passaram do grau leve a moderado $(23,75 \%)$ para a não percepção do handicap $(8,75 \%)$. Dessa forma, apesar de não haver diferença significante entre os grupos, observouse melhora na auto-percepção do handicap nos idosos do Grupo Experimental, sendo esta possivelmente decorrente do TAF.

Em um estudo com programa de orientações durante o processo de adaptação( ${ }^{(25)}$ e outro que aplicou TAF em ido$\operatorname{sos}^{(26)}$, apesar de observarem diminuição na auto-percepção do handicap após o treinamento, também não encontraram diferenças significantes entre os grupos controle e experimental no questionário HHIE. 
Ao final do treinamento auditivo, os idosos do Grupo Experimental estavam fazendo uso de suas próteses auditivas, em período integral e utilizando-as em diferentes ambientes de escuta. Além disso, relataram que se sentiam mais seguros em situações de comunicação difíceis. Quando questionados sobre os efeitos do treinamento auditivo, os idosos não souberam referir com precisão o que havia se modificado, mas a maioria destes estava mais satisfeita com suas próteses auditivas, referindo maior tolerância ao uso da amplificação em locais ruidosos e melhora da atenção auditiva.

Assim sendo, a melhora na qualidade de vida, referida informalmente pelos idosos do Grupo Experimental (com treinamento), pode ser efeito do programa de TAF e aos momentos de aconselhamento semanais, após o término das sessões de estimulação auditiva. Os programas de reabilitação aural devem incluir sessões de aconselhamento, principalmente durante os primeiros 30 dias de uso das próteses auditivas, pois neste período, os pacientes irão decidir se continuarão ou não com o uso das mesmas ${ }^{(27)}$.

A partir dos resultados deste programa, pode-se concluir que o TAF deve ser indicado para todos os idosos usuários de amplificação, pois é possível aprimorar as habilidades auditivas e maximizar os benefícios oferecidos pelas próteses auditivas. Assim sendo, é recomendável que em idosos recém adaptados à amplificação, o TAF deva fazer parte de um programa amplo de reabilitação, que vise também reduzir as dificuldades e inseguranças encontradas nas primeiras semanas de uso.

Em busca de evidências da eficácia do treinamento auditivo formal, a metodologia deste estudo incluiu grupo controle, processo de randomização e estudo duplo-cego. Observações rigorosas dos princípios de pesquisa auxiliam na obtenção de importantes respostas sobre a efetividade do TAF.

\section{CONCLUSÃO}

Diante dos dados apresentados, pode-se concluir que um programa de reabilitação aural, contendo treinamento auditivo formal, auxilia os idosos no período de adaptação das próteses auditivas, bem como modifica o comportamento auditivo destes indivíduos.

\begin{abstract}
Purpose: To investigate the efficiency of a formal auditory training program in hearing aid wearers during the acclimatization period. Methods: Eighteen subjects (mean age of 71.38 years old), male and female, binaural intra-aural hearing aid users for one week participated in the study. Experimental (with auditory training) and Control (without training) groups were formed randomly. Subjects in the experimental group have undergone a formal auditory training program organized in seven 50-minute sessions, once a week. Evaluation procedures included speech recognition tests and a self-assessment questionnaire. These procedures were carried out before ( $1^{\text {st }}$ evaluation $)$ and after $\left(2^{\text {nd }}\right.$ evaluation) the training for the Experimental Group and in the Initial and Final evaluation for the Control Group. Results: In the experimental group, both the word recognition score (WRS) and the speech in noise test showed significantly improved results after the training ( $2^{\text {nd }}$ evaluation). In the study of signal-to-noise ratio for sentence recognition in noise, there was a slight tendency (p-value near 0,05 ) of improvement in the evaluation after the training. It was also observed that for the Experimental Group the results in the $2^{\text {nd }}$ evaluation have not revealed differences in comparison to the results of the Control Group in all tests. Conclusion: It could be concluded that the aural rehabilitation program, including formal auditory training beneficiates the elderly in the period of hearing aids adaptation and also modifies the auditory behavior of these subjects.
\end{abstract}

Keywords: Hearing loss/rehabilitation; Cochlear implants; Hearing aids; Adaptation, physiological/physiology; Aged

\title{
REFERÊNCIAS
}

1. Bess FH, Heddley-Williams A, Lichtenstein MJ. Avaliação audiológica dos idosos. In: Musiek FE, Rintelmann WF. Perspectivas atuais em avaliação auditiva. Barueri: Manole; 2001. p. 343-69.

2. Marques ACO, Kozlowski L, Marques JM. Reabilitação auditiva no idoso. Rev Bras Otorrinolaringol. 2004;70(6):806-11.

3. Russo ICP. Reabilitação da deficiência auditiva no idoso. In: Campos $\mathrm{CAH}$, Costa HOO, editores. Tratado de otorrinolaringologia. São Paulo: Roca; 2002. p. 256-67.

4. Gatehouse S. The time course and magnitude of perceptual acclimatization to frequency responses: evidence from monaural fitting of hearing aids. J Acoust Soc Am. 1992;92(3):1258-68.

5. Bentler R, Holte L, Turner C. An update on the acclimatization issue. Hear J. 1999;52(11):44-7.

6. Cox RM, Alexander GC, Taylor IM, Gray GA. Benefit acclimatization in elderly hearing aid users. J Am Acad Audiol. 1996;7(6):428-41.
7. Humes LE, Wilson DL, Barlow NN, Garner C. Changes in hearing-aid benefit following 1 or 2 years of hearing-aid use by older adults. J Speech Lang Hear Res. 2002;45(4):772-82.

8. Philibert B, Collet L, Vesson J, Veuillet E. The auditory acclimatization effect in sensorineural hearing-impaired listeners: evidence for functional plasticity. Hear Res. 2005;205(1-2):131-42.

9. Verschuure J, van Benthem PP. Effect of hearing aids on speech perception in noisy situations. Audiology. 1992;31(4):205-21.

10. Tuner CW, Humes LE, Benther RA, Cox RM. A review of past research on changes in hearing aid benefit over time. Ear Hear. 1996;17(3 Suppl):14S-25S. Comment in: Ear Hear. 1996;17(3 Suppl):25S-26S. Ear Hear. 1996;17(3 Suppl):26S-28S.

11. Saunders GH, Cienkowski KM. Acclimatization to hearing aids. Ear Hear. 1997;18(2):129-39.

12. Myers S, Palmer CV. Functional and subjective changes over 16 weeks after hearing aid fitting: a case study. Semin Hear. 2005;26(3):140-3. 
13. Robinson K, Summerfield AQ. Adult auditory learning and training. Ear Hear. 1996;17(3 Suppl):51S-65S.

14. Kraus N. Speech-sound perceptual learning. Hear J. 1999;52(11):646.

15. Davis H, Silverman SR, editors. Hearing and deafness. 4th ed. New York: Rinehart and Winston; 1970.

16. Gil D. Treinamento auditivo formal em adultos com deficiência auditiva. [Tese Doutorado]. São Paulo: Universidade Federal de São Paulo. Escola Paulista de Medicina; 2006.

17. Ziliotto ZN, Pereira LD. Estimulação auditiva em cabina acústica: relato de caso. In: Pereira, LD, Azevedo MF, Machado LP, Ziliotto KN. Processamento auditivo: terapia fonoaudiológica. Uma abordagem de reabilitação. São Paulo: Lovise. 2007 no prelo.

18. Pereira LD, Schochat E. Processamento auditivo central: manual de avaliação. São Paulo: Lovise; 1997. 231 p.

19. Burk MH, Humes LE, Amos NE, Strauser LE. Effect of training on word-recognition performance in noise for young normal-hearing and older hearing-impaired listeners. Ear Hear. 2006;27(3):263-78.

20. Tremblay KL. Central auditory plasticity: implications for auditory rehabilitation. Hear J. 2003;56(1):10-5.
21. Hayes EA, Warrier CM, Nicol TG, Zecker SG, Kraus N. Neural plasticity following auditory training in children with learning problems. Clin Neurophysiol. 2003;114(4):673-84.

22. Kricos PB, Holmes AE. Efficacy of audiologic rehabilitation for older adults. J Am Acad Audiol. 1996;7(4):219-29.

23. Kennedy-Vosu L. Hearing loss causes psychological and physical impairment unless detected early. Audiology Alert [serial on the Internet] 2002 [cited 2007 June 12];1(2):[about 7p.] Available from: http://www.audiologycentre.com/audiology_alert2.htm

24. Merzenich M, Pandya P, Tremblay KL. Roundtable discussion: plasticity and auditory training. Semin Hear. 2005;26(3):144-8.

25. Souza AEN, Russo ICP. Um programa de reabilitação audiológica para idosos, novos usuários de aparelho de amplificação sonora individual. Pró-Fono. 1998;10(2):16-22.

26. Megale RL. Treinamento auditivo: avaliação do benefício em idosos usuários de próteses auditivas [tese]. São Paulo: Faculdade de Medicina da Universidade de São Paulo; 2006.

27. Chisolm TH, Willott JF, Lister JJ. The aging auditory system: anatomic and physiologic changes and implications for rehabilitation. Int $\mathrm{J}$ Audiol. 2003;42 (Suppl 2):2S3-10. 